This item was submitted to Loughborough's Research Repository by the author.

Items in Figshare are protected by copyright, with all rights reserved, unless otherwise indicated.

\title{
Robust control of uncertain nonlinear systems: a nonlinear DOBC approach
}

\section{PLEASE CITE THE PUBLISHED VERSION}

http://dx.doi.org/10.1115/1.4033018

\section{PUBLISHER}

(C) ASME

\section{VERSION}

AM (Accepted Manuscript)

\section{PUBLISHER STATEMENT}

This work is made available according to the conditions of the Creative Commons Attribution-NonCommercialNoDerivatives 4.0 International (CC BY-NC-ND 4.0) licence. Full details of this licence are available at: https://creativecommons.org/licenses/by-nc-nd/4.0/

\section{LICENCE}

CC BY-NC-ND 4.0

\section{REPOSITORY RECORD}

Chen, Wen-Hua, Jun Yang, and Zhenhua Zhao. 2016. "Robust Control of Uncertain Nonlinear Systems: A Nonlinear DOBC Approach”. figshare. https://hdl.handle.net/2134/20588. 


\title{
Robust Control of Uncertain Nonlinear Systems: A Nonlinear DOBC Approach
}

\author{
Wen-Hua Chen \\ Department of Aeronautical \\ and Automotive Engineering, \\ Loughborough University, \\ Leicestershire LE11 3TU, UK \\ Email: W.Chen@lboro.ac.uk \\ Jun Yang, Zhenhua Zhao \\ School of Automation, \\ Southeast University, \\ Nanjing 210096, China \\ Email: j.yang84@seu.edu.cn; hndcdfzzh@163.com
}

\begin{abstract}
This paper advocates disturbance observer based control (DOBC) for uncertain nonlinear systems. Within this framework, a nonlinear controller is designed based on the nominal model in the absence of disturbance and uncertainty where the main design specifications are to stabilize the system and achieve good tracking performance. Then a nonlinear disturbance observer is designed to not only estimate external disturbance but also system uncertainty/unmodelled dynamics. With described uncertainty, rigorous stability analysis of the closed-loop system under the composite controller is established in this paper. Finally, the robust control problems of a missile roll stabilization and a mass spring system are addressed to illustrative the distinct features of the nonlinear DOBC approach.
\end{abstract}

\section{Introduction}

Robust control of uncertain nonlinear systems is deemed as one of the most crucial topics in modern control theory [1-3]. High-gain feedback domination technique is a major design tool utilized for robust suppression of uncertainties in nonlinear control theory [3]. However, it is generally claimed that most of existing robust control approaches are criticized as worst case based design, that is, the objective of the controller design is to achieve best performance in the presence of the 
worst uncertainties $[4,5]$. Such a design philosophy involving in most of the existing robust control design methodologies would result in the following two major obstacles for its practical applications [6]. On the one hand, excessive control energy is usually required for the robust control to suppress the worst case uncertainties [5]. This would cause the waste of control energy as well as unreasonable selection of the actuator since powerful actuators are required to perform unnecessarily excessive control action demanded by the robust controllers. To this end, the conventional robust controller in practical engineering usually leads to input saturation problems [7-9]. On the other hand, the robustness performance of those controllers are mostly obtained at a price of sacrificing the nominal control performance of the system because the control performance in the nominal case is usually not considered in the objective function of robust control [4, 6]. However, most practical systems are generally working around its nominal operation point and have a limited chance to operate far away from their nominal operation point. Taking a flight control system as an example, the flight envelop of the vehicle is generally around its nominal flight conditions. The extremely severe flight environment will but rarely appear in the whole flight process. The worst case based robust control design results in over conservative design. As such, a promising and practical robust controller should consider not only the robustness against the worst case of uncertainties but also the control performance around the nominal operation points.

Disturbance observer based control (DOBC) provides an alternative approach for robust control of nonlinear systems [10-13]. In the framework of nonlinear DOBC, the total uncertainties including external disturbances and internal model uncertainties of nonlinear systems are estimated online and then compensated within the closed-loop systems. As a practical robust control approach, nonlinear DOBC has been utilized for uncertainty compensation in engineering practices, for example, see altitude control of missile [14], speed regulation of motor drives [15, 16], tracking control of helicopter [18], DC-bus voltage control of microgrid [19], and control of haptic feedback system [17] . A remarkable superiority of DOBC against most of the existing robust control lies in that it is not a worst case based design approach. In the context of DOBC, a disturbance observer acting as a patch on the baseline controller is employed to estimate the uncertainties. In the absence of uncertainties, the disturbance observer will not be activated and a baseline feedback controller maintains the nominal control performances. In the presence of uncertainties, the disturbance observer is activated for uncertainty estimation and compensation, which achieves the robustness against uncertainties without involving excessive control energy. In general, the DOBC framework facilitates a good trade off between the nominal performance and robustness.

Stability of nonlinear DOBC for nonlinear systems subject to various disturbances (e.g. unknown constant [10], generated by an exogenous system [12] and with bounded variation rate) has been well established. However, it is not applicable when DOBC is used as a robust control method to deal with uncertainties. In this case, different from disturbance, the influence of the uncertainties is actually a function of system states. As such, in spite of promising properties as demonstrated in a number of applications and growing interests in DOBC, the rigorous qualitative robustness analysis on theoretical side lags far behind. It is not reasonable to ignore the coupling between the system dynamics and the observer dynamics when investigating its stability. Recently, in the field of active disturbance rejection control known as another kind of disturbance estimator based control, the robustness stability of the closed-loop systems under uncertainties are established but in the context of linear nominal systems [5] and lower-triangular nonlinear systems [20].

The major contribution of this paper is to develop a general framework for establishing rigorous robust stability for 
uncertain nonlinear systems under the nonlinear DOBC approach. The nominal dynamics of the uncertain nonlinear systems under consideration are nonlinear, which is different from [5, 20]. The Lyapunov stability theory is employed for stability derivation and the results show that the exponentially stability of the uncertain nonlinear system under nonlinear DOBC is guaranteed under certain condition. Simulation studies on a missile roll stabilization and a mass spring system are conducted to show the effectiveness of the nonlinear DOBC approach. The relationship between the disturbance observer gains and quantity of uncertainties are further explored via simulation studies.

\section{Problem Formulation}

In the presence of uncertainty and disturbance, a class of uncertain nonlinear systems may be represented as

$$
\dot{x}=f(x)+\triangle f(x)+[g(x)+\triangle g(x)] u+g_{1}(x) w
$$

where $x \in R^{n}, f(x), g(x)$ are smooth nonlinear functions, $w \in R^{m}$ is the external disturbance, $\triangle f(x)$ and $\triangle g(x)$ are the system uncertainties, and $u \in R^{m}$ is the control input.

It is assumed that the influence of the uncertainty and disturbance can be equivalent to the input channel of the nonlinear system

$$
\dot{x}=f(x)+g(x)[\triangle(x, u, w)+u]
$$

where $g(x) \triangle(x, u, w)=\triangle f(x)+\triangle g(x) u+g_{1}(x) w$. That is, the disturbance and uncertainties satisfy the so called matching condition.

In the context of DOBC approach, the influence of the uncertainty and the disturbance are lumped together and a nonlinear disturbance observer is designed to estimate them. Letting $d=\triangle(x, u, w)$, the system (2) becomes

$$
\dot{x}=f(x)+g(x)(d+u)
$$

In the presence of the matched disturbance, the control $u$ in the $\mathrm{DOBC}$ is proposed in the following form

$$
u=\alpha(x)-\hat{d}
$$

where $\alpha(x)$ is designed by any nonlinear control method based on the nominal plant, i.e. $f(x)$. Normally, it is designed to achieve stability and tracking/regulation specifications. The estimate $\hat{d}$ of the uncertainties is utilized to compensate the influence of lumped uncertainties $d$. In this paper, the nonlinear disturbance observer proposed in $[10,12]$ is adopted to estimate the influence of uncertainties

$$
\left\{\begin{array}{l}
\hat{d}=\xi+p(x), \\
\dot{\xi}=-\gamma l(x) g(x) \xi-\gamma l(x)[f(x)+g(x) u+g(x) p(x)]
\end{array}\right.
$$


where $p(x)$ and $l(x)$ satisfy the relationship

$$
\frac{\partial p(x)}{\partial x}=\gamma l(x)
$$

and $\gamma$ is a positive scalar to be designed. The block diagram of the nonlinear DOBC (4) for uncertain nonlinear system (1) is shown by Fig. 1.

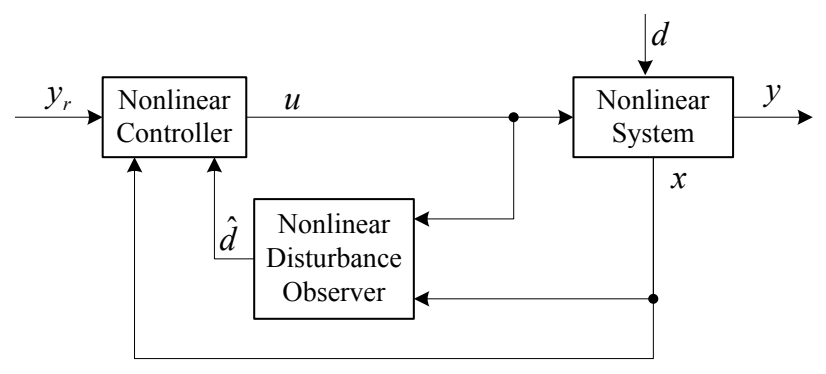

Fig. 1. Block diagram of nonlinear DOBC.

As discussed above, in most cases, DOBC is regarded as a robust control method due to its promising robustness. As an alternative methodology to robust control, DOBC can alleviate some shortcomings (most notably, worst case based design) in robust control. This can be explained by using the basic diagram of DOBC as in Fig. 1 under the assumption of no external disturbance. The inner disturbance observer loop acts as an adaption mechanism in the presence of uncertainty. Rather than directly estimating the uncertain parameters as in many adaptive control algorithms [21], as shown in Eq. (2), it estimates the total difference between the nominal model and the physical system, which may include both structured or unstructured uncertainty. So it is a "crude" adaptive control mechanism. Although it might not achieve the same control performance as other adaptive control algorithms when the uncertainty is structured (e.g. represented by linear functions of unknown parameters), it is more robust than most of adaptive control algorithms. On the other side, as the inner loop is not activated when there is no difference between the nominal model and the physical system, the nominal tracking and regulation performance is maintained. In the presence of uncertainty, the inner disturbance observer loop is activated to estimate and attenuate the influence of the uncertainty. Compared with robust control methods based on the worst case design, the robustness of DOBC may be not as good as them as these methods are designed to achieve best possible performance in the presence of the worst uncertainty. So in the sense, DOBC can be regarded as a "refined" robust control method. However, it provides a promise approach for trading off between the nominal performance and robustness. In summary, DOBC provides an alternative approach to widely used robust control and adaptive control methods for dealing with uncertain (linear or nonlinear) systems.

Stability and performance of the DOBC under various external disturbances have been investigated in [10, 12]. However, so far there is no rigorous stability and performance analysis when the DOBC is applied to deal with uncertainties. This paper focuses on the stability analysis of DOBC under uncertainties. To this end, in the following of this paper, only the influence of the uncertainties is considered, i.e. $d=\triangle(x)$. The key challenge in investigating the stability of DOBC is, different from 
the external disturbance which is independent of the system, the influence of the uncertainty depends on the state of the nonlinear system.

\section{Main Results}

Define the estimation error

$$
e_{d}=d-\hat{d}
$$

Substituting the nonlinear controller (4) and the nonlinear disturbance observer (5) into the uncertain system (3) gives the closed-loop system

$$
\left\{\begin{array}{l}
\dot{x}=f(x)+g(x) \alpha(x)+g(x) e_{d} \\
\dot{e}_{d}=-\gamma l(x) g(x) e_{d}+\dot{d}
\end{array}\right.
$$

Let

$$
\dot{x}=f_{c}(x)=f(x)+g(x) \alpha(x)
$$

be the nominal closed-loop dynamics. Based on the definition of $d$, the dynamics of $d$ can be represented by

$$
\begin{aligned}
\dot{d} & =\frac{\partial \triangle}{\partial x} \dot{x} \\
& =\frac{\partial \triangle}{\partial x}\left(f_{c}(x)+g(x) e_{d}\right)=L_{f_{c}} \triangle(x)+L_{g} \triangle(x) e_{d}
\end{aligned}
$$

Inserting Eq. (10) into Eq. (8) obtains

$$
\left\{\begin{array}{l}
\dot{x}=f_{c}(x)+g(x) e_{d}, \\
\dot{e}_{d}=\left[-\gamma l(x) g(x)+L_{g} \triangle(x)\right] e_{d}+L_{f_{c}} \triangle(x),
\end{array}\right.
$$

We will provide the stability proof for the proposed approach. To this end, Lemma 1 will be firstly introduced.

Lemma 1 [22] (see p. 368) Consider the nonlinear system $\dot{x}=\psi(x, \alpha)$, where $\psi(x, \alpha)$ is continuously differentiable and the Jocobian matrices satisfying $\left\|\frac{\partial \psi(x, \alpha)}{\partial x}\right\| \leq L_{1}$ and $\left\|\frac{\partial \psi(x, \alpha)}{\partial \alpha}\right\| \leq L_{2}\|x\|$ for all $(x, \alpha) \in D \times \Gamma, D \triangleq\left\{x \in R^{n} \mid\|x\|<r\right\}$. Let $k, \lambda, r_{0}$ be positive constant with $r_{0}<r / k$ and define $D_{0}=\left\{x \in R^{n} \mid\|x\|<r_{0}\right\}$. Assume that the trajectory of the system satisfies $\|x(t)\| \leq k\|x(0)\| e^{-\lambda t}, \forall x(0) \in D_{0}, \alpha \in \Gamma, t \geq 0$. Then there is a function $V: D_{0} \times \Gamma \rightarrow R$ such that the following conditions hold

$$
\begin{gathered}
b_{1}\|x\|^{2} \leq V(x, \alpha) \leq b_{2}\|x\|^{2}, \\
\frac{\partial V}{\partial x} \psi(x, \alpha) \leq-b_{3}\|x\|^{2}, \\
\left\|\frac{\partial V}{\partial x}\right\| \leq b_{4}\|x\|,\left\|\frac{\partial V}{\partial \alpha}\right\| \leq b_{5}\|x\|^{2} .
\end{gathered}
$$


where $b_{i}(i=1, \cdots, 5)$ is positive constant. Moreover, if all the assumptions hold globally (in $\left.x\right)$, then $V(x, \alpha)$ is defined and satisfies the above conditions on $R^{n} \times \Gamma$.

In this paper, the following assumptions are imposed to the nonlinear system (1) and the nominal controller in (4):

(A1) $\triangle(0)=0$.

(A2) $f, g, \alpha, \triangle, \frac{\partial \triangle}{\partial x}$ are all continuously differentiable.

(A3) $f_{c}(0)=0, \dot{x}=f_{c}(x)$ is exponentially stable.

Assumption $\mathrm{A} 1$ is for the purpose of convenience and not necessary. As a matter of fact, if $\triangle(0) \neq 0$, the uncertainties $\triangle(x)$ can be separated into two parts as $\triangle(x)=\bar{\triangle}(x)+\triangle(0)$ with $\bar{\triangle}(x)=\triangle(x)-\triangle(0)$ and $\bar{\triangle}(0)=0$. In this case, only the term $\bar{\triangle}(x)$ has to be considered and the term $\triangle(0)$ can be simply merged into the external disturbance term. Assumption A2 is a necessary condition for robustness analysis of DOBC when tackling uncertainties, which has also been employed in related disturbance estimation based control for uncertainties compensation, for example see [5, 20]. Assumption A3 is a basic condition that shall be fulfilled by all successful nonlinear control design.

Theorem 1. Suppose that Assumptions A1-A3 are satisfied. The closed-loop system (11) under the proposed controller (4) and (5) is exponentially stable if the observer gain $l(x)$ is selected such that $\dot{e}_{d}=-\gamma l(x) g(x) e_{d}$ is exponentially stable for all $x \in B_{r} \triangleq\{x \mid\|x\|<r\}$, where $\gamma>0$ is parameter that can be chosen sufficiently large.

Proof: Define a Lyapunov function candidate

$$
V_{0}\left(e_{d}\right)=e_{d}^{2} / 2
$$

for the observer error dynamics in (11). Taking the derivative of $V_{0}\left(e_{d}\right)$ along the dynamics (11) gives

$$
\begin{aligned}
\dot{V}_{0}\left(e_{d}\right) & =\left[-\gamma l(x) g(x)+L_{g} \triangle(x)\right] e_{d}^{2}+L_{f_{c}} \triangle(x) e_{d}, \\
& \leq-\varepsilon e_{d}^{2}+\left|e_{d}\right| \cdot\left|L_{f_{c}} \triangle(x)\right|,
\end{aligned}
$$

where $\varepsilon>0$ and $\gamma$ are chosen such that

$$
\gamma \geq\left(\max _{x \in B_{r}}\left|L_{g} \triangle(x)\right|+\varepsilon\right) / \min _{x \in B_{r}} l(x) g(x) .
$$

It further follows from (12) that

$$
\dot{V}_{0}\left(e_{d}\right) \leq-\varepsilon(1-\theta) e_{d}^{2}, \forall\left|e_{d}\right| \geq \frac{\left|L_{f_{c}} \Delta(x)\right|}{\varepsilon \theta},
$$

where $0<\theta<1$, which shows that the disturbance estimation error dynamics are input-to-state stable by taking $L_{f_{c}} \triangle(x)$ as an input and $e_{d}$ as a state. As such, the response of the disturbance estimation error at an initial state $e_{d}(0)=e_{d}^{0}$ satisfies $e_{d}(t) \in B_{\rho}$ and

$$
\rho=\max \left\{\lambda_{1}\left|e_{d}^{0}\right|, \lambda_{2} \max _{x \in B_{r}}\left|L_{f_{c}} \triangle(x)\right|\right\}
$$


where $\lambda_{1}$ and $\lambda_{2}$ are positive constants.

For the reduced-order nominal system $\dot{x}=f_{c}(x)$, it follows from Assumption A3 that there exists a Lyapunov function $V(x)$ such that [22]

$$
\begin{gathered}
c_{1}\|x\|^{2} \leq V(x) \leq c_{2}\|x\|^{2}, \\
\frac{\partial V}{\partial x} f_{c}(x) \leq-c_{3}\|x\|^{2}, \\
\left\|\frac{\partial V}{\partial x}\right\| \leq c_{4}\|x\| .
\end{gathered}
$$

for all $x \in B_{r_{0}}, r_{0} \leq r$.

The exponential stability of the nonlinear disturbance observer regardless of $x$ (that is, $\dot{e}_{d}=-\gamma l(x) g(x) e_{d}$ is exponentially stable) implies that there exists a $k>0$ and $\lambda>0$ such that the trajectory of the observer error dynamic system satisfies $\left\|e_{d}(t)\right\| \leq k\left\|e_{d}(0)\right\| e^{-\lambda t}, \forall e_{d}(0) \in D_{0}, x \in \Gamma, t \geq 0$ where $D_{0}=\left\{e_{d} \in R^{n} \mid\left\|e_{d}\right\|<\rho_{0}\right\}$ with $\rho_{0}<\rho / k$. It follows from Lemma 1 that there exists a function $W\left(e_{d}, x\right): D_{0} \times \Gamma \rightarrow R$ such that

$$
\begin{gathered}
a_{1}\left\|e_{d}\right\|^{2} \leq W\left(e_{d}, x\right) \leq a_{2}\left\|e_{d}\right\|^{2}, \\
\frac{\partial W}{\partial e_{d}}\left(-l(x) g(x) e_{d}\right) \leq-a_{3}\left\|e_{d}\right\|^{2}, \\
\left\|\frac{\partial W}{\partial e_{d}}\right\| \leq a_{4}\left\|e_{d}\right\|,\left\|\frac{\partial W}{\partial x}\right\| \leq a_{5}\left\|e_{d}\right\|^{2} .
\end{gathered}
$$

for all $e_{d} \in D_{0}$.

Define a Lyapunov function candidate $\Phi\left(x, e_{d}\right)$ for closed-loop system (11) as

$$
\Phi\left(x, e_{d}\right)=V(x)+W\left(e_{d}, x\right) .
$$

Taking the derivative of $\Phi\left(x, e_{d}\right)$ along the trajectory of the closed-loop system (11), one can obtain

$$
\begin{aligned}
\dot{\Phi}= & \frac{\partial V}{\partial x} \dot{x}+\frac{\partial W}{\partial x} \dot{x}+\frac{\partial W}{\partial e_{d}} \dot{e}_{d} \\
= & \frac{\partial V}{\partial x}\left(f_{c}(x)+g(x) e_{d}\right)+\frac{\partial W}{\partial x}\left(f_{c}(x)+g(x) e_{d}\right) \\
& +\frac{\partial W}{\partial e_{d}}\left(\left[-\gamma l(x) g(x)+L_{g} \triangle(x)\right] e_{d}+L_{f_{c}} \triangle(x)\right) \\
\leq & -c_{3}\|x\|^{2}+\left(\frac{\partial V}{\partial x}+\frac{\partial W}{\partial x}\right) g(x) e_{d}+\frac{\partial W}{\partial x} f_{c}(x)-\gamma a_{3}\left\|e_{d}\right\|^{2} \\
& +\frac{\partial W}{\partial e_{d}} L_{g} \triangle(x) e_{d}+\frac{\partial W}{\partial e_{d}} L_{f_{c}} \triangle(x) .
\end{aligned}
$$

In addition, based on Eqs. (15) and (16), we can obtain the following inequalities.

$$
\begin{aligned}
\left(\frac{\partial V}{\partial x}+\frac{\partial W}{\partial x}\right) g(x) e_{d} & \leq\left(\left\|\frac{\partial V}{\partial x}\right\|+\left\|\frac{\partial W}{\partial x}\right\|\right)\|g(x)\| \cdot\left\|e_{d}\right\| \\
& \leq\left(c_{4}\|x\|+a_{5}\left\|e_{d}\right\|^{2}\right)\|g(x)\| \cdot\left\|e_{d}\right\| \\
& \leq c_{4}\|x\| G\left\|e_{d}\right\|+a_{5}\left\|e_{d}\right\|^{3} G
\end{aligned}
$$


where $G \triangleq \max _{x \in B_{r}}\|g(x)\|$. The third term of the right hand side of (17) is estimated as

$$
\begin{aligned}
\frac{\partial W}{\partial x} f_{c}(x) & \leq\left\|\frac{\partial W}{\partial x}\right\| \cdot\left\|f_{c}(x)\right\| \\
& \leq a_{5}\left\|e_{d}\right\|^{2} \cdot L_{3}\|x\| \\
& =d_{1}\left\|e_{d}\right\|^{2}\|x\|
\end{aligned}
$$

where $L_{3}=\max _{x \in B_{r}}\left\|\frac{\partial f_{c}(x)}{\partial x}\right\|$ and $d_{1}=a_{5} L_{3}$. Based on Eq. (16), it is obtained that

$$
\begin{aligned}
\frac{\partial W}{\partial e_{d}} L_{g} \triangle(x) e_{d} & \leq\left\|\frac{\partial W}{\partial e_{d}}\right\| \cdot\left\|L_{g} \triangle(x)\right\| \cdot\left\|e_{d}\right\| \\
& \leq a_{4}\left\|e_{d}\right\| \cdot L_{1} \cdot\left\|e_{d}\right\| \\
& \leq a_{6}\left\|e_{d}\right\|^{2}
\end{aligned}
$$

where $L_{1}=\max _{x \in B_{r}}\left\|L_{g} \triangle(x)\right\|$ and $a_{6}=a_{4} L_{1}$. Based on similarly reason, we can also obtain

$$
\begin{aligned}
\frac{\partial W}{\partial e_{d}} L_{f_{c}} \triangle(x) & \leq\left\|\frac{\partial W}{\partial e_{d}}\right\| \cdot\left\|\frac{\partial \triangle(x)}{\partial x}\right\| \cdot\left\|f_{c}(x)\right\| \\
& \leq a_{4}\left\|e_{d}\right\| \cdot L_{2} \cdot L_{3}\|x\| \\
& \leq a_{7}\left\|e_{d}\right\| \cdot\|x\|
\end{aligned}
$$

where $L_{2}=\max _{x \in B_{r}}\left\|\frac{\partial \triangle(x)}{\partial x}\right\|$ and $a_{7}=a_{4} L_{1} L_{3}$.

Inserting Eqs. (18)-(21) into (17) obtains

$$
\begin{aligned}
\dot{\Phi}\left(x, e_{d}\right) \leq & -c_{3}\|x\|^{2}+c_{4} G\|x\| \cdot\left\|e_{d}\right\|+a_{5} G\left\|e_{d}\right\|^{3} \\
& +d_{1}\left\|e_{d}\right\|^{2} \cdot\|x\|-\gamma a_{3}\left\|e_{d}\right\|^{2}+a_{6}\left\|e_{d}\right\|^{2}+a_{7}\left\|e_{d}\right\| \cdot\|x\|
\end{aligned}
$$

For all $e_{d} \in B_{\rho_{0}}, d_{1}\left\|e_{d}\right\|^{2}\|x\| \leq \tilde{d}_{1}\left\|e_{d}\right\|\|x\|, a_{5} G\left\|e_{d}\right\|^{3} \leq \tilde{a}_{5} G\left\|e_{d}\right\|^{2}$, where $\tilde{d}_{1}=d_{1} \rho_{0}$ and $\tilde{a}_{5}=a_{5} \rho_{0}$. Then Eq. (22) satisfies

$$
\begin{aligned}
\dot{\Phi}\left(x, e_{d}\right) \leq & -c_{3}\|x\|^{2}+\left(c_{4} G+a_{7}+\tilde{d}_{1}\right)\|x\| \cdot\left\|e_{d}\right\| \\
& +\left(-\gamma a_{3}+\tilde{a}_{5} G+a_{6}\right)\left\|e_{d}\right\|^{2} \\
\leq & -c_{3}\|x\|^{2}+\frac{c_{3}}{2}\|x\|^{2}+c_{8}\left\|e_{d}\right\|^{2}+\left(-\gamma a_{3}+\tilde{a}_{5} G+a_{6}\right)\left\|e_{d}\right\|^{2} \\
= & -\frac{c_{3}}{2}\|x\|^{2}-\left(\gamma a_{3}-\tilde{a}_{5} G-a_{6}-c_{8}\right)\left\|e_{d}\right\|^{2}
\end{aligned}
$$

where $c_{8}=\left(c_{4} G+a_{7}+\tilde{d}_{1}\right)^{2} /\left(2 c_{3}\right)$. This implies that the exponential stability of the closed-loop system under the proposed controller is guaranteed by choosing a sufficiently large $\gamma$ such that

$$
\gamma>\left(\tilde{a}_{5} G+a_{6}+c_{8}\right) / a_{3}
$$

A general design procedure of nonlinear DOBC for robust control of nonlinear system is summarized as follows:

Step 1) Design a baseline nonlinear controller $u_{b}=\alpha(x)$ for the nominal system (1) in the absence of disturbances and uncertainties to obtain satisfied stability and other performance specifications. 
Step 2) Design a nonlinear disturbance observer to estimate the disturbances and uncertainties online. Select the observer gain $l(x)$ such that the observer error dynamics is exponentially stable. Tune the observer scalar $\gamma$ to achieve satisfactory estimation accuracy.

Step 3) Integrate the baseline nonlinear control with the disturbance compensation raised by nonlinear disturbance observer to formulate the composite control law.

\section{Simulation Studies}

\subsection{Roll Stabilization for Precision Guided Munitions Class Missiles}

The roll stabilization for PGM (precision guided munitions) class missiles is investigated to illustrate the proposed method in this paper. The dynamics of a PGM clsass missile in roll plane, ignoring the flexible mode, is described as follows [23]

$$
\ddot{\phi}=C_{\alpha} \sin 4 \phi-w_{R R} \dot{\phi}+K_{\delta} \delta_{a}+\delta_{f} C_{\alpha} \sin 4 \phi
$$

where $\phi$ and $\dot{\phi}$ is roll angle and roll rate of PGM missiles, $\delta_{a}$ is fin deflection; $C_{\alpha}, w_{R R}$, and $K_{\delta}$ is the nominal disturbance coefficient, roll rate bandwith and fin effectiveness; $\delta_{f}$ is parameter perturbation coefficient of nominal disturbance. The nominal parameters are chosen as $C_{\alpha}=70, w_{R R}=5$ and $K_{\delta}=1450$. Letting $x_{1}=\phi, x_{2}=\dot{\phi}$ and $u=\delta_{a}$, the dynamic model (24) can be written in state-space form as

$$
\begin{aligned}
& \dot{x}_{1}=x_{2} \\
& \dot{x}_{2}=C_{\alpha} \sin 4 x_{1}-w_{R R} x_{2}+K_{\delta} u+\delta_{f} C_{\alpha} \sin 4 x_{1}
\end{aligned}
$$

The nonlinear disturbance observer for system (25) is designed as:

$$
\begin{aligned}
& \hat{d}=\xi+\gamma x_{2} \\
& \dot{\xi}=-\gamma \xi-\gamma\left(C_{\alpha} \sin 4 x_{1}-w_{R R} x_{2}+K_{\delta} u+\gamma x_{2}\right)
\end{aligned}
$$

The baseline nonlinear controller for system (25) is designed as

$$
\alpha(x)=-\frac{1}{K_{\delta}}\left(C_{\alpha} \sin 4 x_{1}-w_{R R} x_{2}+K_{P} x_{1}+K_{D} x_{2}\right)
$$

Parameters $K_{P}, K_{D}$ are the controller gains and designed as $K_{P}=225$, and $K_{D}=15$. The nonlinear DOBC for system (25) is finally designed as

$$
u=\alpha(x)-\frac{1}{K_{\delta}} \hat{d}
$$

The following simulation scenarios as listed in Table 1 are implemented to illustrate the main results of this paper. 
Table 1. Simulation Scenarios Setting of Roll Stabilization

\begin{tabular}{l|l|l}
\hline \hline Case I & $\gamma=10$ & $\delta_{f 1}=0.5, \delta_{f 2}=1, \delta_{f 3}=1.8$ \\
\hline Case II & $\delta_{f}=0.9$ & $\gamma_{1}=1, \gamma_{2}=10, \gamma_{3}=100$ \\
\hline \hline
\end{tabular}

\subsubsection{Robustness of DOBC with Determined Observer Parameters}

Firstly, we will investigate the robustness of the DOBC with fixed controller and observer parameters. This simulation scenario setting is based on the consideration that the $\mathrm{DOBC}$ with fixed control parameters in general have limited robustness performances, that is, the closed-loop system with fix control parameters possibly becomes unstable in the presence of some extremely severe uncertainties. The robustness of the DOBC with observer scalar $\gamma=10$ is investigated under different parameter perturbation coefficients: $\delta_{f 1}=0.5, \delta_{f 2}=1, \delta_{f 3}=1.8$ in this subsection. The response curves of roll angle, roll rate, and fin deflection under DOBC with $\gamma=10$ in the presence of uncertainties of Case $I$ are shown by Figs. 2.

As shown by Fig. 2, the DOBC with given control parameters could effectively compensate the plant uncertainties of $\delta_{f_{1}}=0.5$ and $\delta_{f_{2}}=1$. However, the closed-loop system becomes unstable once the uncertainty is up to $\delta_{f_{3}}=1.8$. As a result, it is concluded from the above analysis that the DOBC with determined parameters have limited robustness performances. In the next subsection, we will investigate how to tune the parameters of DOBC so that the closed-loop system could cover a larger stability region.

\subsubsection{Robustness of DOBC with Different Observer Scalars}

In this subsection, the simulation studies are conducted for the roll stabilization for PGM class missiles with fixed uncertainties of Case II: $\delta_{f}=0.9$ under DOBC with different observer scalars. The purpose of those simulation studies is to show how the observer scalar $\gamma$ can be tuned to achieve better robustness and performances in the presence of uncertainties. The variable responses of the roll stabilization system under DOBC in the presence of uncertainties described by Case II are shown by Figs. 3.

In the presence of uncertainty $\delta_{f}=0.9$, it can be observed from Fig. 3 that the closed-loop system is unstable with an observer scalar $\gamma=1$. By increasing the observer scalar to $\gamma=10$, it is shown by Fig. 3 that the output of closed-loop system converges to its desired reference asymptotically. As shown by Fig. 3, the variable response curves of the roll stabilization system under DOBC with an observer scalar $\gamma=100$ is much closer to the nominal control system, indicating a much better transient control performance, which is referred to as transient performance recovery in [2]. In conclusion, the simulation results in this subsection reveal that a larger observer scalar generally indicates a larger stability region, which confirms the theoretic results presented in Section 3. 


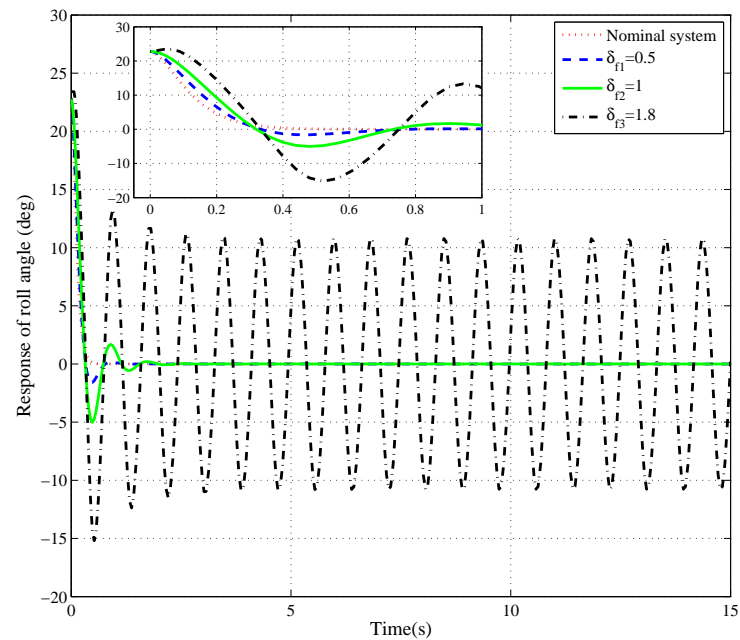

(a)

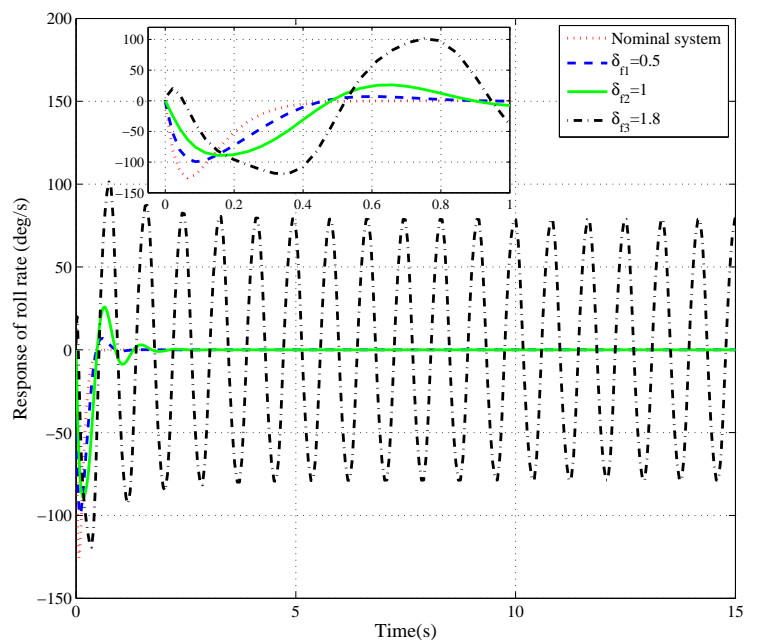

(b)

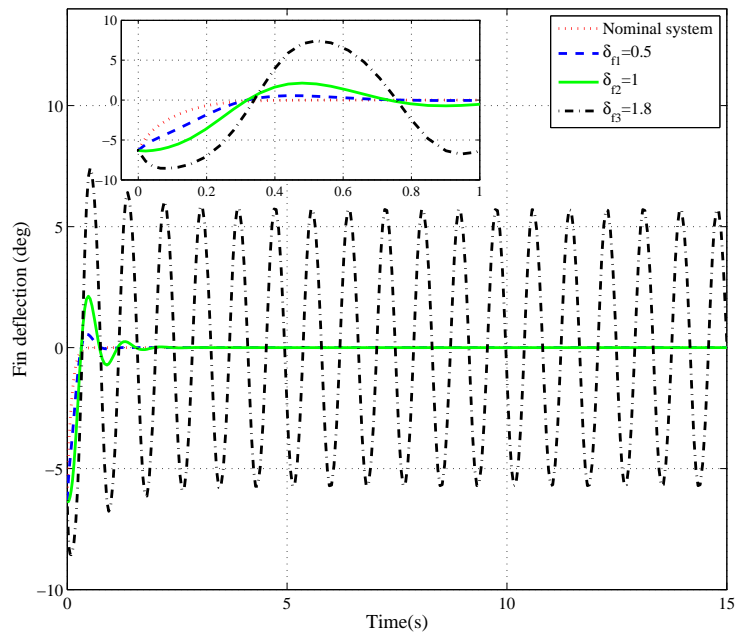

(c)

Fig. 2. Response curves of the roll stabilization system (25) under DOBC (28) with $\gamma=10$ in the presence of various cases of uncertainties $\delta_{f}$ (Case I): (a) roll angle, (b) roll rate, (c) fin deflection. 


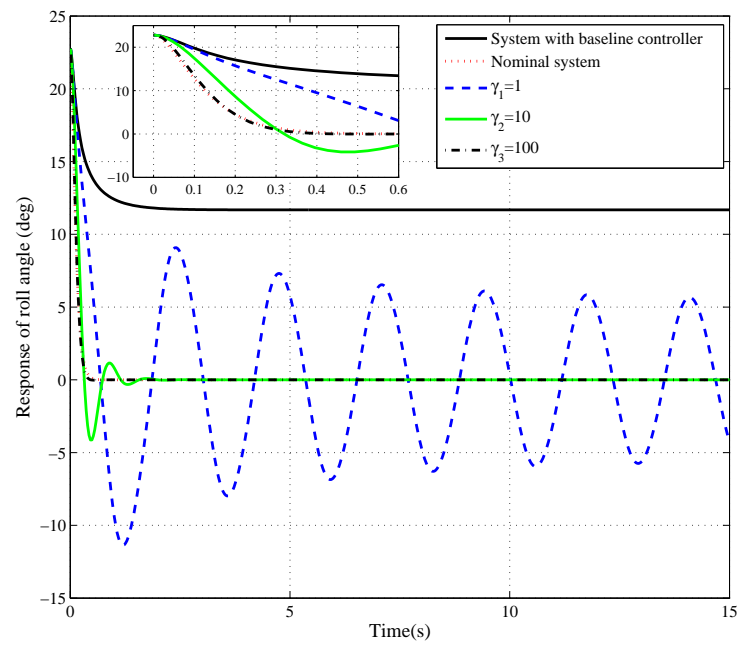

(a)

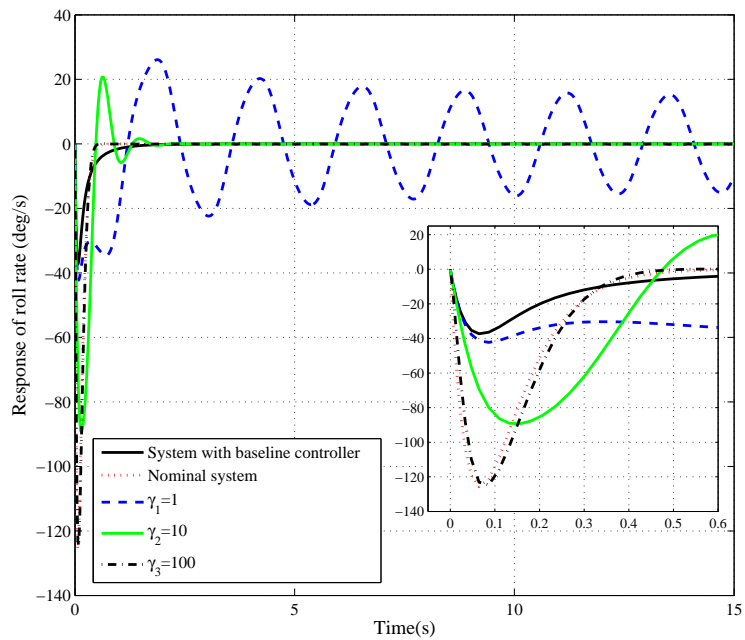

(b)

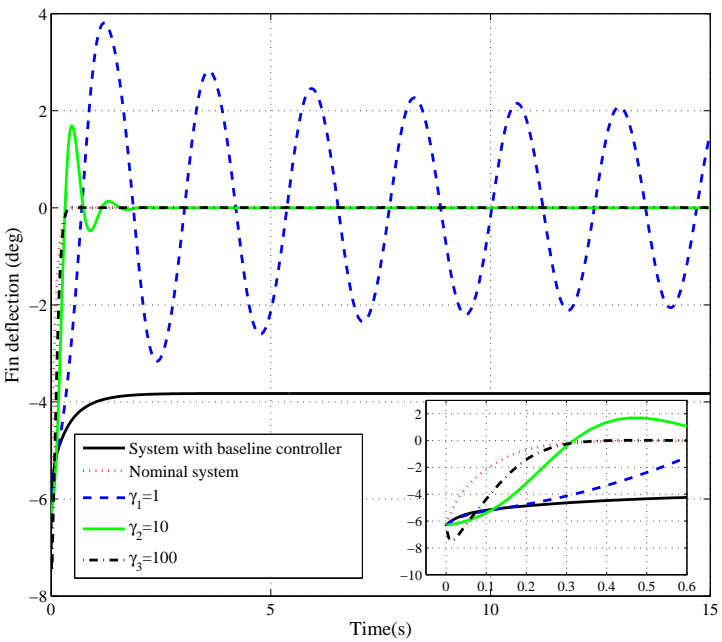

(c)

Fig. 3. Response curves of the roll stabilization system (25) under DOBC (28) with various observer scalar $\gamma$ in the presence of uncertainties $\delta_{f}=0.9$ (Case II): (a) roll angle, (b) roll rate, (c) fin deflection. 


\subsection{Displacement Tracking for Nonlinear Spring Mechanical System}

A mechanical system with a spring of nonlinear dynamics as shown in Fig. 4 is introduced to illustrate the proposed method in this paper. The dynamical equation of the system is described as follows [24, 25]

$$
m \ddot{x}+c \dot{x}+f_{1} x+f_{3} x^{3}=k_{t} u
$$

where $x, u, m, c$ and $k_{t}$ are the displacement, controller force, mass, damping and the torque constant, respectively. $f_{1}$ and $f_{3}$ are the parameters to describe the characteristics of the nonlinear spring. The dynamic model (29) can be written in

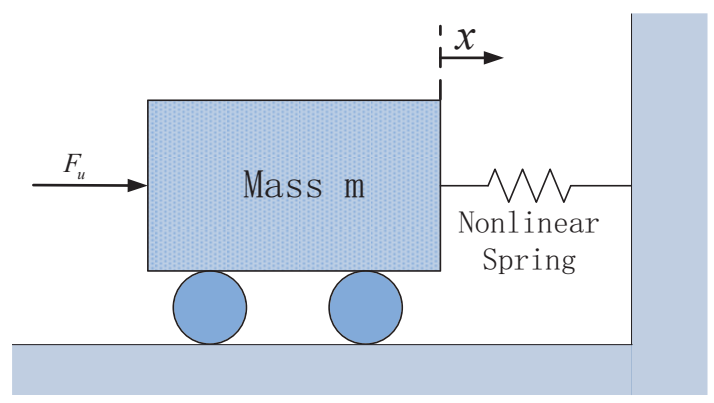

Fig. 4. Mass spring system

state-space form as

$$
\begin{aligned}
& \dot{x}_{1}=x_{2} \\
& \dot{x}_{2}=-\frac{c}{m} x_{2}-\frac{f_{1}}{m} x_{1}-\frac{f_{3}}{m} x_{1}^{3}+\frac{k_{t}}{m} u
\end{aligned}
$$

where $x_{1}, x_{2}$ and $u$ are the displacement, velocity and controller force, respectively. There always exists some uncertainties in the parameters of the nonlinear spring. Let $\delta_{f_{1}}=\frac{f_{1}-f_{10}}{f_{1}}$ and $\delta_{f_{3}}=\frac{f_{3}-f_{30}}{f_{3}}$ represent the uncertain parts of $f_{1}$ and $f_{3}$, where $f_{10}$ and $f_{30}$ denote the nominal values of $f_{1}$ and $f_{3}$, respectively. The nominal parameters are referred to $[24,25]$ and listed in Table 2.

\begin{tabular}{c|ccccc} 
Table 2. Nominal Parameters of the Mass Spring System \\
\hline \hline Parameter & $m$ & $c$ & $f_{10}$ & $f_{30}$ & $k_{t}$ \\
\hline Unit & $\mathrm{kg}$ & $N s / m$ & $N / m$ & $N / m^{3}$ & 1 \\
\hline Value & 1 & 5 & 100 & 500000 & 1 \\
\hline \hline
\end{tabular}


The nonlinear disturbance observer for system (30) is designed as:

$$
\begin{aligned}
& \hat{d}=\xi+\frac{\gamma m}{k_{t}} x_{1} \\
& \dot{\xi}=-\gamma \xi-\gamma\left(u+\frac{\gamma m}{k_{t}} x_{1}\right)+\frac{\gamma}{k_{t}}\left(c x_{2}+f_{10} x_{1}+f_{20} x_{1}^{3}\right)
\end{aligned}
$$

The baseline nonlinear controller for system (30) is designed as

$$
\alpha(x)=\frac{1}{k_{t}}\left(c x_{2}+f_{1} x_{10}+f_{30} x_{1}^{3}\right)+\frac{m}{k_{t}}\left[-K_{P}\left(x_{1}-x_{1 d}\right)-K_{D} x_{2}-K_{I} \int_{0}^{t}\left(x_{1}-x_{1 d}\right) d t\right]
$$

where $x_{1 d}$ is the reference signal of the displacement $x_{1}$. Parameters $K_{P}, K_{I}$ and $K_{D}$ are the controller gains to be determined, which are designed as $K_{P}=48, K_{I}=64$ and $K_{D}=12$, respectively. The desired reference of displacement is set as $x_{1 d}=0.02$. The nonlinear DOBC for system (30) is finally designed as

$$
u=\alpha(x)-\frac{m}{k_{t}} \hat{d}
$$

\subsubsection{Robustness of DOBC Against Uncertainties}

Table 3. Simulation Scenarios Setting of Robustness Test

\begin{tabular}{l|l|l}
\hline \hline Scenarios & Observer Scalar & Uncertainties \\
\hline Case I & $\gamma=6$ & $\delta_{f_{3}}=0, \delta_{f_{1}}=-40 \%,-84 \%, 84 \%$ \\
\hline Case II & $\gamma=6$ & $\delta_{f_{1}}=0, \delta_{f_{3}}=-8 \%,-13.5 \%, 13.5 \%$ \\
\hline \hline
\end{tabular}

First, we will investigate the robustness of the DOBC against various uncertainties with fixed controller and observer parameters. This simulation scenario setting is based on the consideration that the DOBC with fixed control parameters in general have limited robustness performances, that is, the closed-loop system with fixed control parameters possibly becomes unstable in the presence of some extremely severe uncertainties. The robustness of the DOBC with observer scalar $\gamma=6$ is investigated under two cases of uncertainties (Case I: $\delta_{f_{1}}=-40 \%,-84 \%, 84 \%$ and Case II: $\delta_{f_{3}}=-8 \%,-13.5 \%, 13.5 \%$, as shown by Table 2) in this subsection. The response curves of displacement, velocity and control force under DOBC with $\gamma=6$ in the presence of uncertainties of Case I and Case II are shown by Figs. 5 and 6, respectively.

As shown by Fig. 2, the DOBC with given control parameters could effectively compensate the plant uncertainties of $\delta_{f_{1}}=-40 \%$ and $\delta_{f_{1}}=84 \%$. However, the closed-loop system becomes unstable once the uncertainty is up to $\delta_{f_{1}}=-84 \%$. It is observed from Fig. 3 that the DOBC approach with above parameters has achieved appropriate robustness against the plant uncertainties of $\delta_{f_{3}}=-8 \%$ and $\delta_{f_{3}}=13.5 \%$. When the uncertainty is up to $\delta_{f_{3}}=-13.5 \%$, it can be seen from Fig. 3 that the response curves of the mass spring system under DOBC becomes oscillating. As a result, it is concluded from the above analysis that the DOBC with determined parameters have limited robustness performances. In the next subsection, we will investigate how to tune the parameters of DOBC so that the closed-loop system could cover a larger stability region. 


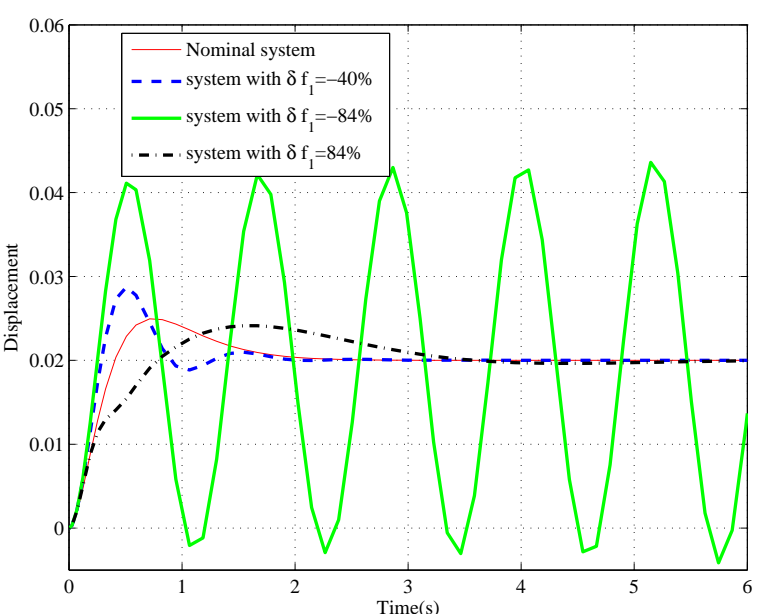

(a)

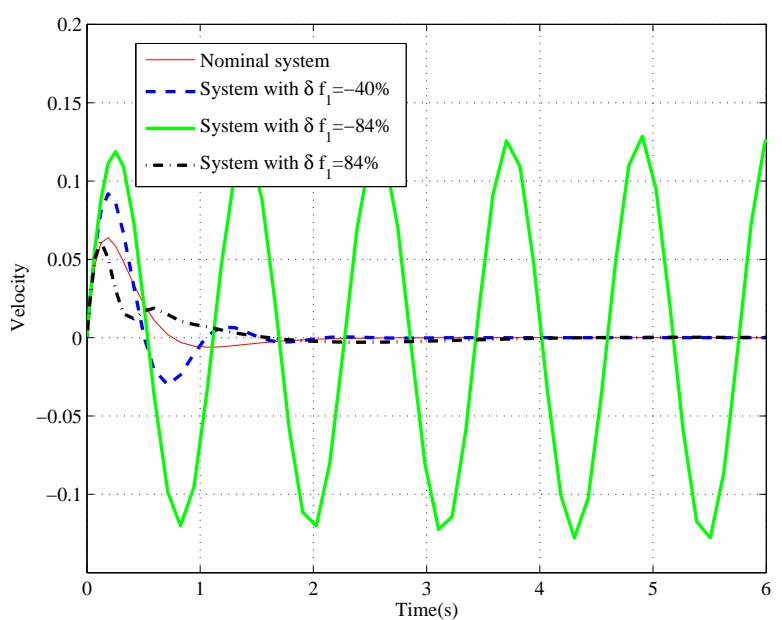

(b)

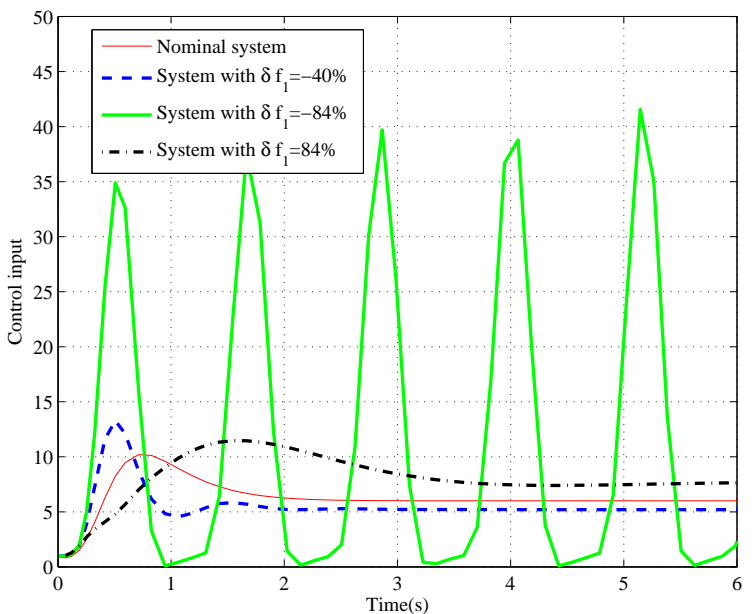

(c)

Fig. 5. Response curves of the mass spring system (29) under DOBC (33) with $\gamma=6$ in the presence of various cases of uncertainties $\delta_{f_{1}}$ (Case I): (a) displacement, (b) velocity, (c) control force. 


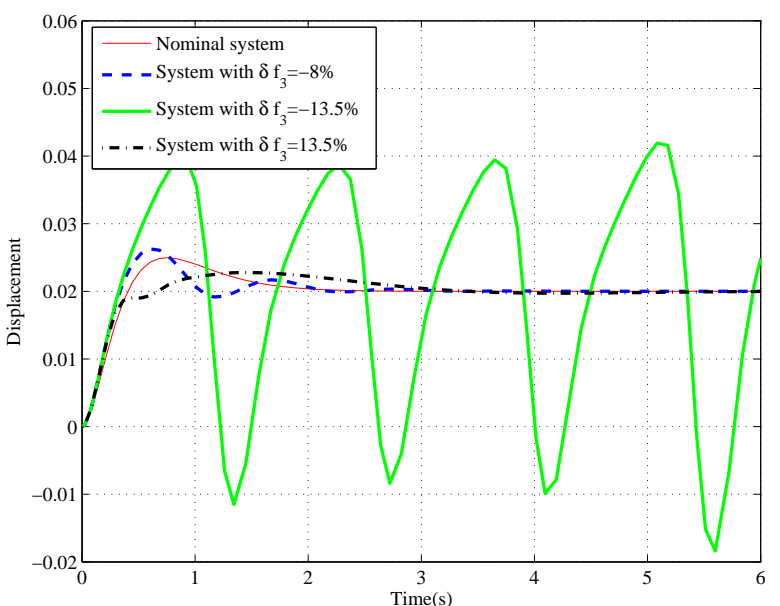

(a)

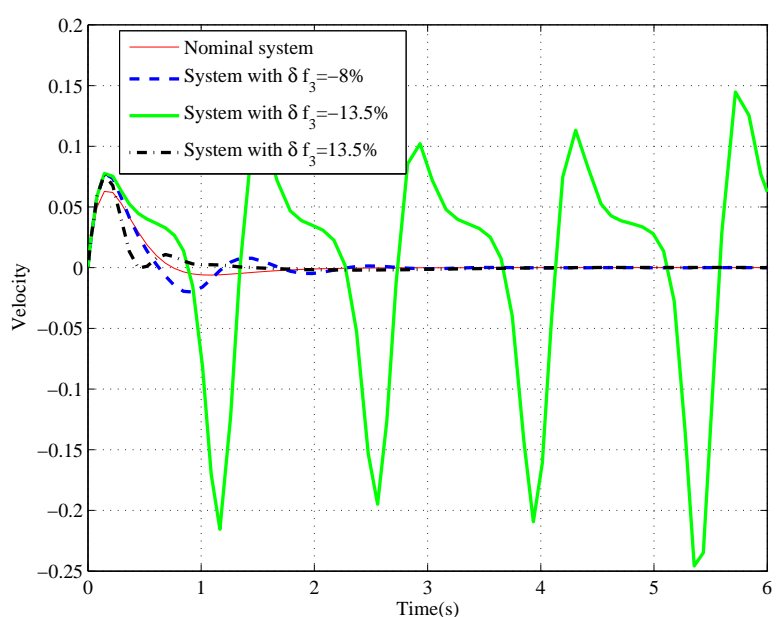

(b)

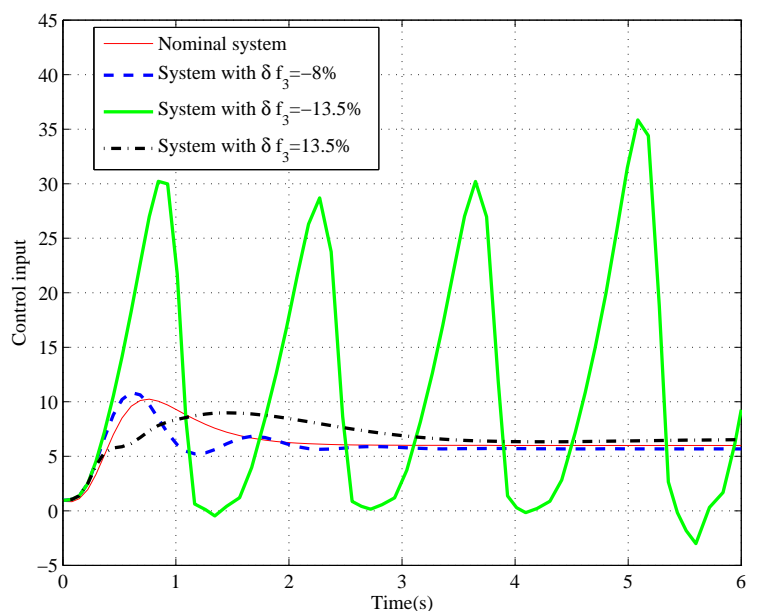

(c)

Fig. 6. Response curves of the mass spring system (29) under DOBC (33) with $\gamma=6$ in the presence of various cases of uncertainties $\delta_{f_{3}}$ (Case II): (a) displacement, (b) velocity, (c) control force. 


\subsubsection{Design Parameter Influence on Robustness of DOBC}

Table 3. Simulation Scenarios Setting of Different DOBC parameters

\begin{tabular}{l|l|l}
\hline \hline Scenarios & Uncertainties & Observer Scalar \\
\hline Case III & $\delta_{f_{1}}=-50 \%, \delta_{f_{3}}=0$ & $\gamma_{1}=1.8, \gamma_{2}=5, \gamma_{3}=20$ \\
\hline Case IV & $\delta_{f_{1}}=0, \delta_{f_{3}}=-30 \%$ & $\gamma_{1}=16.5, \gamma_{2}=22, \gamma_{3}=50$ \\
\hline \hline
\end{tabular}

In this subsection, the simulation studies are conducted for the mass spring system with two cases of fixed uncertainties (Case III: $\delta_{f_{1}}=-50 \%$ and Case IV: $\delta_{f_{3}}=-30 \%$, as shown by Table 3) under DOBC with different observer scalars. The purpose of those simulation studies is to show how the observer scalar $\gamma$ can be tuned to achieve better robustness and performances in the presence of uncertainties. The variable responses of the mass spring system under DOBC in the presence of uncertainties described by Case III and Case IV are shown by Figs. 7 and 8, respectively.

In the presence of uncertainty $\delta_{f_{1}}=-50 \%$, it can be observed from Fig. 7 that the closed-loop system is unstable with an observer scalar $\gamma=1.8$. By increasing the observer scalar to $\gamma=5$, it is shown by Fig. 7 that the output of closed-loop system converges to its desired reference asymptotically. As shown by Fig. 8, further increasing the observer scalar to $\gamma=20$ brings a much better transient tracking performance including smaller tracking errors and shorter settling time. Similarly, for the case of uncertainty $\delta_{f_{3}}=-30 \%$, it is observed from Fig. 8 that the closed-loop system is unstable with a small observer scalar of $\gamma=16.5$. As the scalar increased to $\gamma=22$, it is shown by Fig. 8 that the uncertainty can be effectively compensated from the closed-loop system. As shown by Fig. 8, the variable response curves of the mass spring system under DOBC with an observer scalar $\gamma=50$ is much closer to the nominal control system, indicating a much better transient control performance, which is referred to as transient performance recovery in [2]. In conclusion, the simulation results in this subsection reveal that a larger observer scalar generally indicates a larger stability region, which confirms the theoretic results presented in Section 3.

\section{Conclusions}

Robust control of uncertain nonlinear systems has been investigated in this paper via a nonlinear DOBC approach. Rigorous robust stability analysis of uncertainties in nonlinear systems under nonlinear DOBC is established for matched disturbances and uncertainties. Design guidance of nonlinear DOBC has been suggested with the help of the stability analysis results. It has been shown that for certain given plant uncertainties, the nonlinear DOBC approach could achieve asymptotical stability with certain large observer gain $\gamma$. Simulation examples of roll stabilization for PGM classical missiles and a nonlinear mass spring mechanical system have been investigated to show the effectiveness of the nonlinear DOBC approach and the interaction between the uncertain level and the observer gain in the nonlinear disturbance observer. The simulation results have confirmed the theoretic results presented in the paper. 


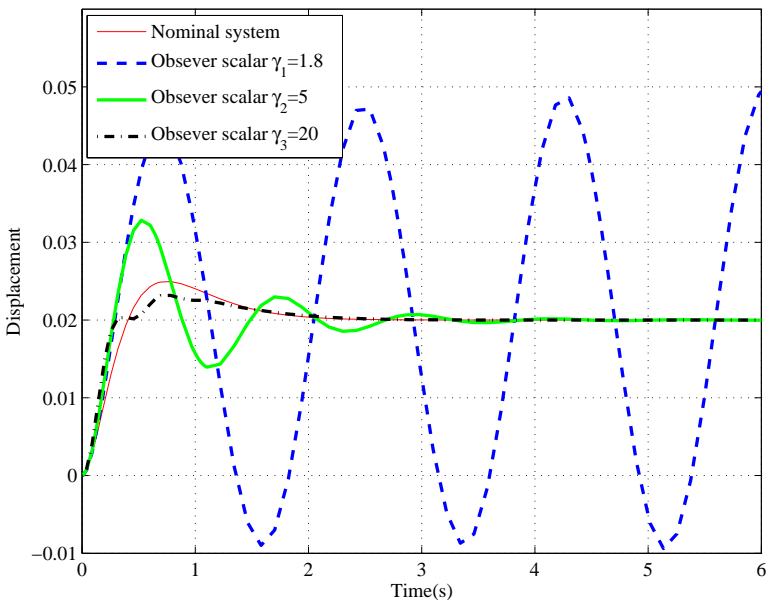

(a)

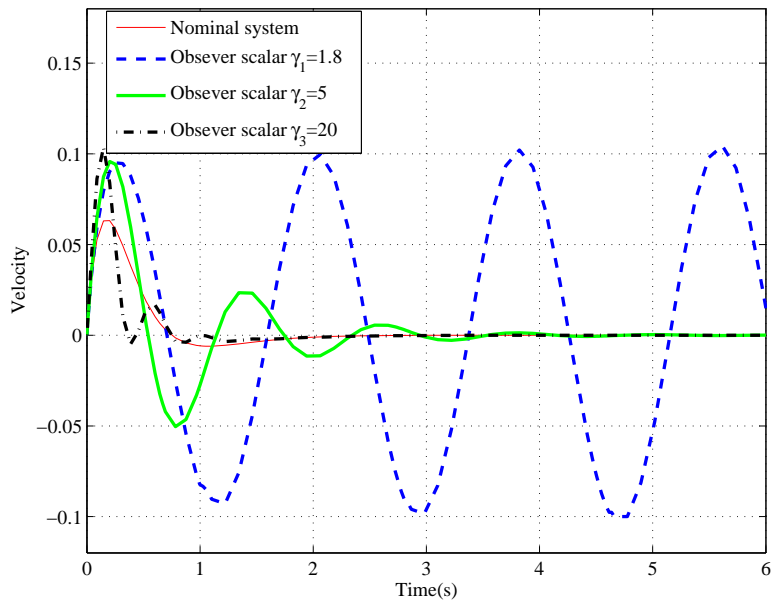

(b)

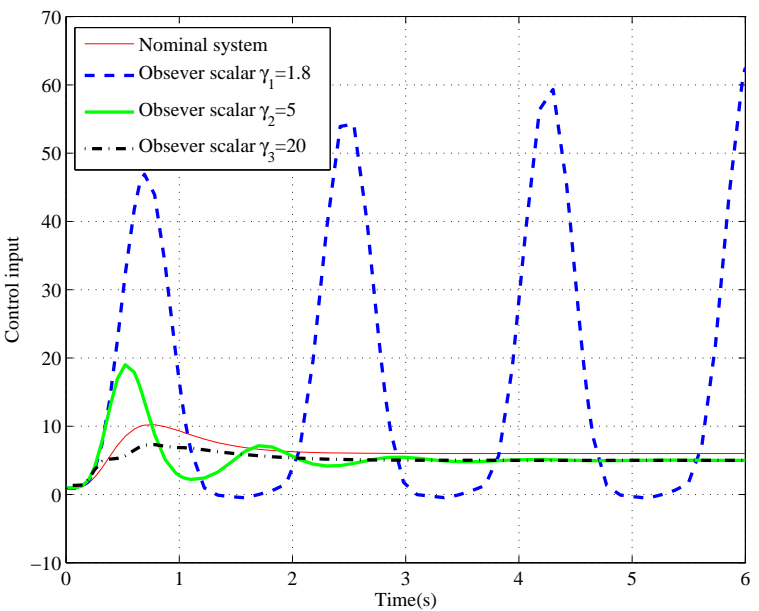

(c)

Fig. 7. Response curves of the mass spring system (29) under DOBC (33) with various observer scalar $\gamma$ in the presence of uncertainties $\delta_{f_{1}}=-50 \%$ (Case III): (a) displacement, (b) velocity, (c) control force. 


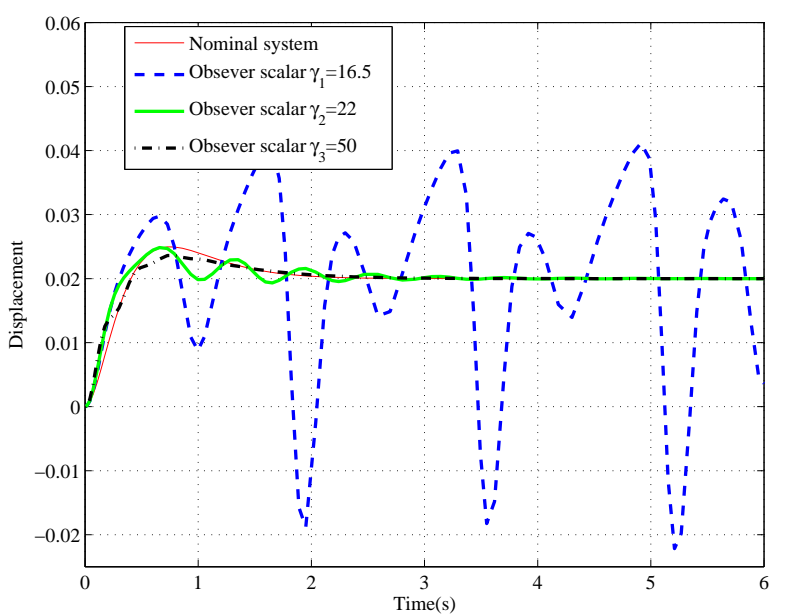

(a)

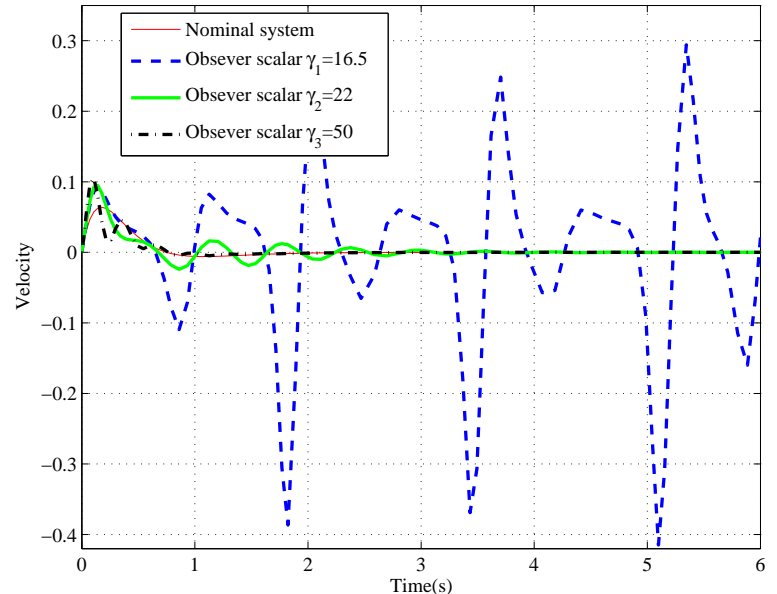

(b)

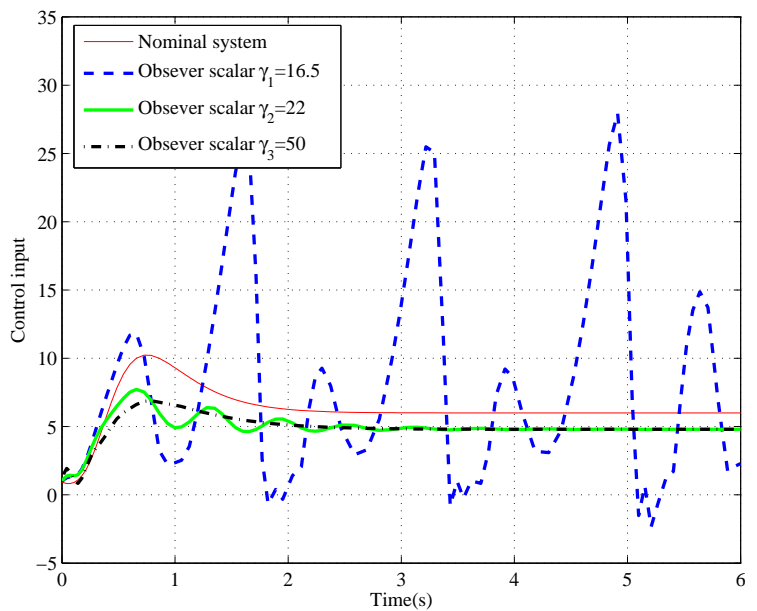

(c)

Fig. 8. Response curves of the mass spring system (29) under DOBC (33) with various observer scalar $\gamma$ in the presence of uncertainties $\delta_{f_{3}}=-30 \%$ (Case IV): (a) displacement, (b) velocity, (c) control force. 


\section{Acknowledgements}

This work was supported in part by the National Natural Science Foundation of China under Grants 61203011 and 61573099, the Natural Science Foundation of Jiangsu Province under Grant BK2012327, Chinese Postdoctorial Science Foundation under Grants 2013M540406 and 2014T70455, and Fundamental Research Funds for the Central Universities.

\section{References}

[1] Atassi, A. N., and Khalil, H. K., 1999, "A separation principle for the stabilization of a class of nonlinear systems," IEEE Trans. Autom. Control, 44, pp. 1672-1687.

[2] Freidovish, L. B., and Khalil, H. K., 2008, "Performance recovery of feedback-linearization based designs," IEEE Trans. Autom. Control, 53, pp. 2324-2334.

[3] Qian, C., and Lin, W., 2002, "Output feedback control of a class of nonlinear systems: a nonseparation principle paradigm,” IEEE Trans. Autom. Control, 47, pp. 1710-1715.

[4] Yang, J., Chen, W.-H., and Li, S., 2011, "Non-linear disturbance observer-based control for systems with mismatched disturbances/uncertainties," IET Control Theory and Application, 5, pp. 2053-2062.

[5] Guo, B.-Z., and Zhao, Z.-L., 2013, “On convergence of the nonlinear active disturbance rejection control for MIMO systems," SIAM Journal of Control and Optimization, 51, pp. 1727-1757.

[6] Li, S., Yang, J., Chen, W.-H., and Chen, X., 2014, Disturbance Observer-Based Control: Methods and Applications, CRC Press, Boca Raton, USA.

[7] Chen, H., 2014, "Robust stabilization for a class of dynamic feedback uncertain nonholonomic mobile robots with input saturation,” International Journal of Control, Automation and Systems, 12, pp. 1216-1224.

[8] Chen, H., Wang, C., Liang, Z., Zhang, D., and Zhang, H., 2014, "Robust Practical Stabilization of Nonholonomic Mobile Robots Based on Visual Servoing Feedback with Inputs Saturation,” Asian Journal of Control, 16, pp. 692702.

[9] Xu, B., Shi, Z., and Yang, C., 2015, "Composite fuzzy control of a class of uncertain nonlinear systems with disturbance observer," Nonlinear Dynamics, 80, pp. 341-351.

[10] Chen, W.-H., Ballance, D. J., Gawthrop, P. J., and O’Reilly, J., 2000, “A nonlinear disturbance observer for robotic manipulators," IEEE Trans. Ind. Electron., 47, pp. 932-938.

[11] Chen, W. H., 2003, "Harmonic disturbance observer for nonlinear systems," Journal of Dynamic Systems, Measurement, and Control, 125, pp.114-117.

[12] Chen, W.-H., 2004, "Disturbance observer based control for nonlinear systems," IEEE/ASME Trans. Mechatron., 9, pp. 706-710.

[13] Chen, W.-H., Yang, J., Guo, L., and Li, S., 2016, "Disturbance observer-based control and related methods: An overview," IEEE Trans. Ind. Electron., 63, pp. 1083-1095.

[14] Chen, W.-H., 2003, “Nonlinear disturbance observer-enhanced dynamic inversion control of missiles,” Journal of Guidance, Control and Dynamics, 26, pp. 161-166.

[15] Yao, J., Jiao, Z., and Ma, D., 2015, “Output feedback robust control of direct current motors with nonlinear friction 
compensation and disturbance rejection,” Journal of Dynamic Systems, Measurement, and Control, 137, 041004.

[16] Errouissi, R., Ouhrouche, M., Chen, W.-H., and Trzynadlowski, A. M., 2012, "Robust cascaded nonlinear predictive control of a permanent magnet synchronous motor with antiwindup compensator,’ IEEE Trans. Ind. Electron., 59, pp. 3078-3088.

[17] Gupta, A., and OMalley, M. K., 2011, "Disturbance-observer-based force estimation for haptic feedback," Journal of Dynamic Systems, Measurement, and Control, 133, 014505.

[18] Liu, C. J., Chen, W.-H., and Andrews, J., 2004, “Tracking control of small-scale helicopters using explicit nonlinear MPC augmented with disturbance observers,” Control Engineering Practice, 3, pp. 258-268.

[19] Wang, C., Li, X., Guo, L., and Li, Y., 2014, "A nonlinear-disturbance-observer-based DC-bus voltage control for a hybrid AC/DC microgrid," IEEE Trans. Power Electron., 29, pp. 6162-6177.

[20] Xue, W., and Huang, Y., 2014, “On performance analysis of ADRC for a class of MIMO lower-triangular nonlinear uncertain systems," ISA Transactions, 53, pp. 955-962.

[21] Yin, C., Cheng, Y., Chen, Y., Stark, B., and Zhong, S., 2015, “Adaptive fractional-order switching-type control method design for 3D fractional-order nonlinear systems," Nonlinear Dynamics, 82, pp. 39-52.

[22] Khalil, H. K., 2002, Nonlinear Systems. 3rd., Prentice hall, Upper Saddle River, USA.

[23] F. W. Nesline, H. B. Wells, and P. Zarchan, 1981. "Combined Optimal/Classical Approach to Robust Missile Autopilot Design,” AIAA Journal of Guidance and Control, 4, pp. 316-322.

[24] Misawa, E. A., 1997, "Discrete-time sliding mode control for nonlinear systems with unmatched uncertainties and uncertain control vector," Journal of Dynamic Systems, Measurement, and Control, 119, pp.503-512.

[25] Chen, W.-H., Ballance, D. J., Gawthrop,P. J., and O'Reilly, J., 1999, "Nonlinear PID predictive controller.” IEE Proceedings-Control Theory and Applications, 146, pp. 603-611. 